\title{
Las familias y los cuidados a las personas mayores dependientes: entre la reciprocidad y la ambivalencia
}

\author{
Families and the Care of Dependent Older People: \\ Between Reciprocity and Ambivalence \\ Concepción RoDRÍGUEz RODRÍGUEZ \\ Consejería de Sanidad y Asuntos Sociales \\ Junta de Comunidades de Castilla-La Mancha \\ concepcionr@jccm.es
}

Recibido: 24/05/2013

Revisado: 27/06/2013

Aceptado: 06/11/2013

Disponible on line: 20/12/2013

\begin{abstract}
Resumen
La atención a las personas mayores dependientes en nuestro contexto social depende fundamentalmente de las familias. Sobre esta realidad se articulan diferentes elementos que dan cuenta de la complejidad de la situación; cuestiones como las representaciones sociales sobre el envejecimiento y la dependencia, los cuidados personales y la construcción de los roles de género, la solidaridad familiar y la responsabilidad pública en la atención. Todo ello se articula con los procesos relacionales que cada familia cuidadora debe afrontar y resolver para hacer frente a la atención de las personas dependientes. La experiencia de trabajo grupal con personas cuidadoras sirve como guía para mostrar parte de estos procesos y dinámicas familiares frente a los cuidados (la redefinición de los vínculos, la ambivalencia entre el deber de cuidar y las aspiraciones, los recursos, y las oportunidades disponibles, la desorientación, la incertidumbre, etc.). Esta formalización no refleja la complejidad del fenómeno del cuidado, mas algunas experiencias como las que se desarrollan en el artículo dan cuenta de las oportunidades de intervención en ámbitos de atención primaria, como recurso de apoyo complementario y de reconocimiento, frente a la ayuda institucional tan escasa que reciben los cuidadores actualmente.
\end{abstract}

Palabras clave: dependencia, personas cuidadoras, relaciones familiares, grupos de apoyo.

\begin{abstract}
The care of dependent older people in our social context depends primarily on families. Within this reality, different elements that account for the complexity of the situation are articulated; issues such as social representations on ageing and dependence, self-care and the construction of gender roles, family solidarity, and public accountability for care. This is all articulated with the relational processes that each caregiver family must confront and resolve in order to address the care of dependent persons. The experience of group work with caregivers serves as a guide to show part of these processes and the family dynamics relative to care (the redefinition of bonds, the ambivalence between the duty of providing care and the aspirations, resources, and opportunities available, disorientation, uncertainty, etc). This formalisation does not reflect the complexity of the phenomenon of providing care, however, experiences of the type developed in this article inform about intervention opportunities in areas of primary care, as a resource of complementary support and recognition, in the face of the scarce institutional support that caregivers currently receive. Keywords: dependence, caregivers, family relationships, support groups.
\end{abstract}

Referencia normalizada: Rodríguez Rodríguez, C. (2013): «Las familias y los cuidados a las personas mayores dependientes: entre la reciprocidad y la ambivalencia». Cuadernos de Trabajo Social, 26(2): 349-358.

Sumario: Introducción. 1. Los cuidados a las personas mayores dependientes y la reciprocidad familiar. 2. El apoyo social: los grupos de ayuda mutua. 3. Elementos de la dinámica relacional familia-anciano dependiente. 4. Referencias bibliográficas. 


\section{Introducción}

La atención y cuidado de las personas mayores, y en concreto de los mayores dependientes, se está convirtiendo en los últimos años en una cuestión de interés, y objeto de análisis, investigación y de planificación de políticas públicas; y la Ley 39/2006 de Promoción de la Autonomía Personal y Atención a las personas en situación de Dependencia es su exponente legal. De este modo, ha pasado de ser una realidad asignada al ámbito privado y de competencia casi exclusiva de las familias, a un asunto público que además se está conformando como un problema social. En este sentido, por un lado se trata de una situación social a la que se debe dar respuestas porque afecta al bienestar y a las oportunidades de desarrollo de personas y familias; pero por otro lado, en la coyuntura socioeconómica actual se plantea cómo se debe responder, cuál debe ser la cobertura de ayuda, cuál el detrimento de recursos, quién debe asumir la responsabilidad, etc. Esta realidad no es ajena a una cierta imagen social de las personas mayores. Imagen que tiene un contenido simbólico y que habla de cómo se valoran y qué se espera de ellas, que nos remite a ideas de déficit, limitaciones, exclusión, desinterés, aislamiento, descalificación, enfermedad y muerte.

Además, como señalan Abellán, Esparza, Castejón y Pérez (2011), el giro de lo privado a lo público,

Resulta de transformaciones que abarcan prácticamente todas las dimensiones del proceso de modernización social: demográfica, epidemiológico-sanitaria, familiar/convivencial, de género, diseño y distribución de los servicios público/privado, progreso social y material, relación estado/mercado/familia, y creciente multidimensionalidad del abordaje con que se entiende y estudia la dependencia (p. 8).

Las investigaciones y los análisis estadísticos muestran la importancia cuantitativa de una realidad que, además de sumamente amplia, es compleja y heterogénea. Se evidencia un escenario con un aumento progresivo de la población mayor, con el incremento de aquella de mayor edad, asociado a mayores tasas de dependencia y necesidades de cuidados de larga duración, con un descenso en la mortalidad y sobre todo un aumento de las enfermedades crónicas frente a las transmisibles. Se constata que al igual que se ha producido un proceso de transición demográfica, se puede hablar también de una transición epidemiológica; las enfermedades infecciosas ya no son la principal causa de discapacidad y muerte, sino que ahora predominan las enfermedades crónicas: enfermedades del aparato circulatorio, cáncer y enfermedades respiratorias, y se destaca el aumento de la mortalidad por enfermedades como la demencia y el Alzhéimer (Carretero, Garcés, Ródenas y Sanjosé 2006).

Estos primeros apuntes perfilan sólo una parte del mapa que se pretende recorrer, porque la realidad que comienza ya a mostrarse invita a hacerse una serie de preguntas que serán el hilo conductor de estas reflexiones: ¿Cómo cuidar de estas personas?, ¿quién las cuida? y ¿en qué se sustenta el cuidado?

\section{Los cuidados a las personas mayores de- pendientes y la reciprocidad familiar}

La dependencia se define como limitaciones o situaciones ligadas a la falta o pérdida de autonomía (física, mental, intelectual o sensorial), de carácter permanente que afectan a las personas y que requieren de atención y cuidados (para la realización de una o varias actividades básicas) de manera prolongada (Ley 39/2006). Por lo tanto dos elementos se distinguen en las situaciones de dependencia: la necesidad de cuidados y ayuda de otros y su duración a lo largo del tiempo, dado el carácter permanente de los estados de dependencia (los denominados cuidados de larga duración). Por otro lado, la diversidad de tipos de ayuda necesarios para atender estas situaciones plantea la responsabilidad y la complejidad que supone la atención; se trata de cuidados de salud o médicos; cuidados personales, de alimentación, tareas domésticas, compras, medicación, transporte, gestiones diarias, así como ayudas técnicas, adaptaciones de la vivienda, etc.

Hasta este momento, a pesar del desarrollo de la Ley indicada y de la extensión de servicios públicos y privados de asistencia personal, residenciales, etc., la realidad que muestran las estadísticas y las diferentes investigaciones realizadas sobre los cuidados de personas dependientes, indican claramente que se prestan principalmente en el entorno familiar. Rogero (2010) afirma: 
La distribución de los cuidadores informales en España refleja la existencia de una jerarquía de cuidadores relacionada con la cercanía de parentesco [...] las relaciones intergeneracionales son primordiales en la atención a mayores en nuestro país, incluso por delante del cuidado de los cónyuges o parejas (p. 199).

Este escenario viene a corroborar la afirmación de Durán (2002) que el peso de los cuidados se encuentra soportado por las familias, en el hogar, y añade que se trata de un ámbito cuyos costes son invisibles para los poderes públicos. Por otro lado, a dicha invisibilidad se une que son básicamente las mujeres quienes prestan dichos cuidados; las tareas del cuidado personal han estado asignadas y asociadas tradicionalmente a la construcción y desarrollo del rol de género, en el ámbito privado y con un escaso reconocimiento social, asignación que probablemente ha determinado la escasa visibilidad y valor asignados a dichas tareas; el Libro Blanco sobre la atención a las personas en situación de dependencia en España (2005) ya situaba en el 84 por ciento el porcentaje de mujeres que eran cuidadoras principales. Así pues, el perfil que surge de los cuidadores informales es que son mayoritariamente mujeres, con una edad media de 50 años y cuya principal ocupación son las tareas domésticas (Laparra, 2001; Casado, García-Gómez, López y Massons, 2007).

La relación que se establece entre responsables del cuidado y personas no ocupadas (jubiladas, desempleadas de una ocupación previa y personas que se dedican a las tareas del hogar) muestra la desigualdad en la responsabilidad de los cuidados. La cuestión que se plantea es: ¿qué relación existe entre hacerse cargo del cuidado de una persona dependiente y las oportunidades de acceso al mercado laboral de la persona cuidadora?, ¿cuál es el coste que tiene la dedicación a las labores de cuidado para las personas cuidadoras, en términos económicos, de ocio, de desarrollo personal, etc.?, ¿cómo se puede visibilizar la carga y la necesidad de apoyos cuando quienes cuidan en su mayoría son personas que o bien ya han abandonado el mercado laboral (jubiladas) o bien su ocupación principal ha sido «similar» a la que desarrollan con los cuidados personales y del hogar?

En cualquier caso, como plantea Bazo (2008), el asunto de los cuidados familiares y de la solidaridad intergeneracional es un debate de interés social, fundamentado en cuestiones como la capacidad y la voluntad de las familias para el cuidado de las generaciones mayores, las expectativas y preferencias que se crean entre las diversas generaciones respecto a los cuidados y el debido equilibrio en la participación entre la familia y el Estado. Dicha solidaridad, que permite la protección familiar, se sustenta en el vínculo afectivo que une a sus miembros y que hace que se procuren cuidados, atenciones y preocupaciones, pero también conlleva un conjunto de obligaciones y de derechos contraídos entre parientes; de tal forma que dar cuidados y prestar ayuda implica, a su vez, el derecho de recibir estos mismos dentro de una red que se establece a lo largo del tiempo y que acaba implicando lealtad y gratitud. Ahora bien, tal principio de reciprocidad no evita que la protección familiar adquiera formas, intensidades y variaciones que dependen de la estructura familiar, las necesidades individuales de sus miembros, la etapa del ciclo familiar, la disponibilidad de recursos, la proximidad o no de los miembros, etc.(Izquieta, 1996).

Hoy día hay un discurso generalizado acerca del debilitamiento de los lazos de protección familiar; la realidad no parece corresponderse con esta imagen, si bien se deben tener en consideración los cambios, las tensiones y dificultades que los vínculos familiares atraviesan y que afectan a la cantidad, calidad y continuidad del apoyo familiar como fuente básica de ayuda entre las personas. Se asiste a una disminución del número de miembros en la familia, que limita la disponibilidad de los cuidadores y la cantidad de cuidados; al alargamiento de la edad de las personas ancianas que conlleva unas necesidades de cuidados, cuyos costes y exigencias (materiales y psicológicas) superan la capacidad de los hijos, quienes además deben responder a sus propias actividades $\mathrm{y}$ requerimientos personales y familiares; los mismos valores sociales de autonomía, independencia o individualismo entran en contradicción con unas necesidades y unos requerimientos de cuidado, compromiso y apoyo; la movilidad geográfica de las personas, la distancia física entre quienes pueden prestar ayuda hacen difícil, si no imposible, cumplir con esta función; y por último, los cambios en el 
rol social de las mujeres, principales protagonistas de los cuidados, como ya se ha señalado (ídem).

\section{El apoyo social: los grupos de ayuda mutua}

Los cuidados en el medio de la persona mayor dependiente es la alternativa más extendida, incluso preferida, por el anciano y su familia, pero no cabe duda de que tiene una serie de implicaciones que afectan a los recursos y las capacidades del entorno de aquél, como se ha mencionado anteriormente. La evidencia es que se trata de cuidados que, en la mayor parte de los casos, se han de dispensar a largo plazo, en los que participa la red del anciano de manera intensiva (son en ocasiones atenciones para todas las actividades básicas e instrumentales de la vida diaria de la persona), imprevisibles en la extensión de su duración y que pueden requerir de adaptaciones constantes. Todo ello provoca situaciones que afectan a la salud física y mental de la persona que cuida, llegándose a conceptualizar como carga del cuidador.

Los recursos de apoyo formal tratan de aliviar y complementar la labor del cuidador, así como mediar sobre el impacto que estas tareas tienen para la vida de quien cuida $y$, en consecuencia, para la persona dependiente. Carretero, Garcés, Ródenas y Sanjosé sostienen que se ha demostrado que la patología de la persona cuidada y la reacción del cuidador frente a los síntomas del dependiente pueden tener un peso similar en el desarrollo de la carga.

Los autores citados sistematizan los tipos de recursos de apoyo social que pueden recibir las personas cuidadoras, diferenciando los servicios de respiro familiar (ayuda domiciliaria, centros de día y respiro residencial o nocturno), y los programas psicoeducativos y psicosociales (grupos de apoyo, de ayuda mutua, de entrenamiento en habilidades cognitivo-conductuales, etcétera).

La experiencia concreta que se analiza en el presente trabajo es la de un grupo de apoyo con cuidadoras de personas mayores dependientes. La intervención grupal tuvo una duración de un año y medio, mediante sesiones quincenales. El rol del profesional fue inicialmente emprender la organización del grupo, que se realizó a través de la coordinación con los Servicios Sociales municipales, quienes con- tactaron con las familias cuidadoras sin definir ningún tipo de sesgo en la selección (en cuanto a sexo, edad, tiempo de cuidado, tipo de patología de la persona cuidada, parentesco, etc.). El grupo se constituyó finalmente con 10 personas, todas ellas mujeres con unas edades comprendidas entre los 30 y los 60 años. En la totalidad de los casos estaban cuidando a sus progenitoras, excepto uno en el que el dependiente era el hermano; el tiempo que llevaban cuidando era muy variable, pero en todos los casos duraba más de dos años.

Los datos que sostienen el presente análisis sobre los procesos relacionales en situaciones de cuidado de personas dependientes se han obtenido de las narraciones y testimonios de las participantes (transcritos inmediatamente finalizada cada sesión) durante el desarrollo de este grupo de apoyo; estas narrativas han sido agrupadas para su análisis en categorías conceptuales que abarcan una gran parte de las características de las dinámicas relacionales que subyacen al cuidado.

Es importante señalar que la organización y desarrollo de este tipo de grupos supone asumir una necesaria postura metodológica e ideológica en la práctica profesional, en la medida que implica un cambio en la concepción de las personas, las relaciones y las situaciones que viven, como claramente expresa Gracia Fuster (1997),

Desde una perspectiva de déficit a una de potencialidades y promoción de habilidades y recursos comunitarios, que incorporen los principios de autodeterminación, autoconfianza y ayuda mutua en el proceso de provisión de ayuda y que promuevan relaciones basadas en la igualdad y en la responsabilidad compartida (p. 38).

Se trata de construir un espacio donde, a través del contacto y el compromiso común entre personas que viven situaciones similares, se puedan crear vínculos de pertenencia, compartir los problemas y las alternativas emprendidas por cada una de ellas. Una de las funciones básicas de este tipo de grupos es evitar el sentimiento de aislamiento y soledad que generan los cuidados continuos; las dificultades materiales, emocionales, relacionales, al ser puestas en común y recibir el feed back del grupo, dejan de ser consecuencia de algún tipo de negli- 
gencia, dificultad o fracaso personal, para pasar a recibir apoyo, aliento, soluciones comunes, y un nuevo modelo de comprensión que ayude a la persona a afrontar los problemas. Problemas que, como señalan Sison, Aparicio y Guinaldo (2012), hacen que «algunas decisiones que desde el punto de vista «práctico» son sencillas, desde el punto de vista emocional pueden llegar a desbordar a los cuidadores y sus familia» (p. 73), y que ponen en cuestión, en ocasiones, sus propias creencias y capacidades.

En la primera sesión se realizó el encuadre básico del grupo: sesiones quincenales de una hora y media de duración; a las participantes se les pidió confidencialidad respecto a lo elaborado en las sesiones, y compromiso en la asistencia, con el fin de garantizar la necesaria confianza entre todas. Se informó que el objetivo era crear un espacio compartido donde poder abordar las dificultades, preocupaciones y temores que viven como cuidadores, así como las soluciones que van poniendo en práctica, y por último, se insistió que no se trataba de un grupo en el que se les iba a enseñar cómo desempeñar mejor su tarea.

Desde el inicio de las sesiones se pone de manifiesto un propósito de las participantes respecto al grupo: la necesidad de «desahogo» para hacer frente a las emociones provocadas por la centralidad de los cuidados en sus vidas, las dudas y las experiencias vividas, con la posibilidad de ayudar a otras personas con realidades similares; pero también el grupo se vive como un reconocimiento a su labor, como un apoyo frente a una situación que afirman viven en soledad.

\subsection{Elementos de la dinámica relacional fa- milia-anciano dependiente}

Las diferentes experiencias narradas y vividas por las cuidadoras del grupo de apoyo permiten comprender, más allá de las estadísticas, la complejidad de los procesos relacionales, los conflictos, los cambios intrafamiliares y la vivencia del cuidado que experimentan estas personas, porque ¿qué pautas de relación familiar se estructuran en torno a los cuidados?, ¿qué desafíos afectan a la dinámica familiar y a su evolución?, ¿cómo influye la historia relacional previa de la familia sobre los cuidados y los vínculos que se organizan en el aquí y en el ahora?, ¿cómo se estructuran los roles en rela- ción al cuidado?, ¿cómo intervienen las creencias y los valores de los miembros de la familia sobre el cuidado, el compromiso con el otro?, ¿cómo afecta el sistema asistencial sobre las decisiones, los cuidados, la aceptación de la enfermedad?

\subsection{Cuidado y crisis familiares}

Camdessus, Bonjean y Spector (1995) plantean un análisis de las crisis que viven las familias con un miembro anciano. El concepto de crisis se define, en los términos de Caillé y Rey (1990), como una pérdida de equilibrio en el sistema, que ve cuestionado su modo de funcionamiento habitual y conocido. Se trata de un cambio en los roles que han sido definidos a lo largo del tiempo tras diferentes negociaciones y conflictos. La familia ahora tiene que cuestionar y renegociar su modo de funcionamiento, incluso modificar sus expectativas mutuas. Cuando un progenitor anciano comienza a mostrar síntomas de demencia, a sufrir pérdidas de autonomía, la familia debe adaptarse a la nueva situación de atención que progresivamente se irá generando, a la vez que el anciano debe integrar esta pérdida de autonomía. La crisis, como se decía, pone en cuestión el modelo: las decisiones que se deben tomar no estaban previstas, obligan a reorganizarse, a veces frente a acontecimientos imprevisibles y desconcertantes (como se escuchó en la primera sesión del grupo); las referencias, las ideas sobre las personas, sus relaciones cambian (quien fue tu padre/madre ahora no te reconoce, pasa a depender de ti, cambia su carácter, y es probable que no sirvan los mismos argumentos para conseguir su entendimiento, su acuerdo o su colaboración).

En la presentación del grupo apareció una primera realidad compartida por la mayoría de participantes: el peor momento vivido como cuidadoras fue el origen, tras el diagnóstico de la enfermedad o la decisión de que la persona mayor ya no podía vivir sola. En la situación de crisis que vivieron, a la confusión experimentada respecto a cómo organizar las tareas de cuidado, se unió la inseguridad sobre cómo actuar, más aún en la medida en que los síntomas de la enfermedad no remitían y la nueva experiencia iba superando los recursos materiales y emocionales de la familia (reorganización de tiempos, espacios, dedicaciones, pero 
también asunción de un cambio en los roles tradicionales y en las relaciones entre el anciano y su familia), apareciendo sentimientos de culpa, desesperación, incertidumbre, etc.

El problema de la crisis es - según Camdessus, Bonjean y Spector-que en ocasiones este cuestionamiento del funcionamiento familiar se vive como una amenaza y puede producir lo que denominan «un deslizamiento de sentido» entre la enfermedad y el envejecimiento; así, si la enfermedad remite a unos síntomas que si fueran tratados podrían mejorar y volver a la situación inicial, el envejecimiento abre a otro mundo de significados que remiten a la pérdida, a la dependencia y, en última instancia, a la muerte; aparece la negación de esta pérdida y se busca la solución a la avería, a la enfermedad. Los autores se preguntan cuántos de los casos de solicitud de ingreso de un anciano en una residencia o un hospital no son acciones movidas por este deslizamiento: "solicito un ingreso porque está enfermo y necesita un tratamiento, aunque sé que nada se puede hacer».

La experiencia de una de las participantes, una mujer joven cuidadora principal de su madre dependiente, diagnosticada con una demencia, puede ayudar a ver este deslizamiento. Ella ha negociado con sus otros dos hermanos que será la cuidadora en su propio domicilio, recibiendo el apoyo intermitente de éstos para su descanso. Su principal propósito es mantener a su madre activa, conectada, y por ello salen a pasear todos los días, le habla continuamente, le pone música - «porque a ella le gustaba cantar»- y baila con ella. El deterioro va apareciendo progresivamente: ya no puede caminar, apenas habla, y sobre todo no puede comer; todo esto lo describe llorando, muy asustada, todos los días pide cita con su médico para buscar una solución que no resuelve nada, unido a los conflictos que le está generando con su esposo e hijos. En una sesión posterior, menos angustiada, cuenta que su madre ha estado en el hospital, donde le han informado que su deterioro es irreversible y le han retirado toda la medicación, manteniendo únicamente pautas de alimentación y cuidados paliativos. Afirma que ya sabe que su madre va a morir, su propósito es que esté lo más cómoda y tranquila posible, además ahora, « puedo entender a mi familia, las cosas que me decían y así me apoyan».
Parece acertado sostener que el esfuerzo de esta cuidadora por buscar desesperadamente soluciones se presentaba como un mecanismo que evitaba o negaba afrontar la inminente pérdida de su madre, una mujer a la que había estado muy unida.

\subsection{Conflicto de lealtades}

Los desafíos que enfrentan las familias de dos o más generaciones ante las situaciones de cuidados y la adaptación a las nuevas demandas requieren la puesta en marcha de patrones de relación flexibles. Se trata de replantear el vínculo entre padres e hijos, de forma que se integren pautas de desligamiento y diferenciación entre ellos, a la vez que un compromiso de continuidad con la relación ante los cambios que afrontan. Es lo que King y Wynne (2004) definen como madurez filial: un tipo de reciprocidad en la que la generación de los hijos habrá aceptado la necesaria ayuda que deben prestar a sus padres ancianos y éstos a su vez, recíprocamente, estarán dispuestos a aceptar el apoyo e intervención de sus hijos.

En esta situación, una revisión de las pautas de apego (Hill, Fonagy, Safier y Sargent, 2003) y cuidado entre hijos y padres frente a la enfermedad y el envejecimiento puede suponer aumentar la proximidad y la involucración en el cuidado, que además se acompaña de una inversión de este vínculo (cuidar a quien te cuidó). Esta nueva aproximación puede ser vivida por los padres como una experiencia penosa, en la medida que sienten que se trata de una pérdida de poder, de responsabilidad o de independencia; no en vano las limitaciones que vive la persona mayor en este momento suponen una pérdida de control tanto sobre su propia capacidad física como sobre su entorno. Es necesario que se negocie esta nueva aproximación sin que resulte limitador para el anciano, aumente o le haga sentir que se intensifica su dependencia, a la vez se deben definir los márgenes de la autonomía de la persona cuidadora, el alcance de su responsabilidad y su capacidad. La dificultad aparece cuando hablar de la enfermedad, los cuidados o la dependencia se convierte en una tarea penosa, temida, porque anticipa un cambio en el ciclo vital, una posible pérdida; se sobrentiende, además, que los padres no tienen ideas acerca de su envejecimiento y su muerte y se cree que no hablar 
sobre lo penoso de la situación les protege (Rolland, 2000).

Esta realidad puede provocar una asunción de responsabilidades en el cuidador que le sobrecarguen, a la vez que fomenten la ambigüedad sobre los cuidados que se ofrecen: $¿$ es esta la situación que el progenitor desearía si pudiese elegir?, ¿se están tomando las mejores decisiones para él?; si todo lo que se hace es para su bienestar ¿por qué razón no responde, no cambia la pauta de su comportamiento, no atiende a razones? (y esto en las familias que cuidan de un anciano con demencia es algo que se escucha con frecuencia). Rolland propone realizar un trabajo de revisión con las familias de los mitos y las creencias sobre el deber de cuidado, la lealtad hacia los progenitores, la experiencia multigeneracional de cuidados en la familia; una revisión que ayude a expresar, cuestione y a la vez facilite elegir qué mantener y qué cambiar de esta herencia.

El relato de otra cuidadora participante en el grupo hace pensar que se encuentra atrapada entre el deber de cuidar y su profundo agotamiento: cada día, desde hace seis años, acude a casa de los padres, recibe además las llamadas continuas del padre pidiéndole ayuda porque la anciana está gritando durante horas, no para de llorar, no le reconoce y está asustada, o ha revuelto toda la casa buscando sus joyas. Refiere que, si le pide a su padre que reconsidere su actitud de enfado frente a estos comportamientos de la mujer, recibe acusaciones de desatención (es que tu sólo vienes un rato, no estás aquí todo el día como yo). Por otro lado, ésta es la segunda fórmula que han buscado para el bienestar de los dos ancianos, ya estuvieron dos años conviviendo todos, la pareja de ancianos y la familia de ella (que a su vez viven en el lugar donde tiene su propio negocio), pero el descontrol de la anciana y el conflicto entre todos los miembros de la familia hacían la situación insostenible. ¿Qué expresó en la primera sesión del grupo? El profundo agradecimiento y deber que tenía hacia esta mujer, quien se casó con su padre viudo y se hizo cargo de ella como si fuera su propia hija, dice; pero la cuestión que se le plantea es: ¿hasta cuándo vas a poder mantener las atenciones y los cuidados? Los sentimientos de responsabilidad y deber se entremezclan con las dudas sobre si esta situación es la mejor pa- ra la anciana, si el padre aguantará más tiempo, o qué ocurrirá si enferma también, entre todo esto aparece la culpa porque en ocasiones, entre el agotamiento y el enfado, piensa que de seguir así será necesario tomar otra decisión.

\subsection{Renegociando relaciones}

El problema en esta nueva etapa del ciclo vital familiar puede surgir, afirma Wynne (1991), cuando «la calidad del trato es inapropiada o proporciona un ajuste deficiente entre las personas en la época en la que intervienen estos cambios» (p. 146). No se trata sólo de acompasar los procesos del ciclo de desarrollo de cada uno de los sistemas que forman la familia (son sistemas complejos porque integran una variedad de miembros en su composición y por tanto se entremezclan diferentes vínculos), sino que además se trata de sistemas con historia, que han ido creando su propia trama de relaciones y vínculos a lo largo del tiempo y que ahora se pueden poner en cuestión. Se produce un solapamiento de procesos entre la enfermedad (o las necesidades de atención que tiene la persona dependiente), el sistema familiar y los individuos de lo componen (Navarro, 2004).

En la situación de la primera cuidadora aludida, ante los nuevos cambios que la enfermedad provocaba en su madre, multitud de intenciones y necesidades se le entremezclaban: necesitaba evitar y frenar el deterioro, devolver a su madre todo lo recibido de ella, eludir la pérdida inminente, pero además se sentía en medio de una familia que demandaba atenciones (la acusaba de un cierto abandono y cuestionaba su habilidad como cuidadora). Muchas son las cuestiones que surgen en estas situaciones: ¿a quién deben atender?, ¿por qué, en ocasiones, las familias no acompañan en las necesidades de las cuidadoras en estos conflictos de lealtades a los que se enfrentan?, ¿qué les impide comunicarse en términos de comprensión y no de reproche?, ¿por qué las cuidadoras no pueden escuchar, ni dejarse aconsejar por la familia o emplear un recurso de respiro? Las cuidadoras viven un conflicto de lealtades entre las necesidades de los dependientes y las demandas de su familia nuclear, con el añadido de culpa que esto le acarreaba.

La mutualidad es el cuarto componente epigenético de los procesos relacionales familiares que propone Wynne, la pauta relacional que 
permitirá a las familias poder resolver y acordar los cambios que afectan a su organización frente a los nuevos acontecimientos que se les imponen. La mutualidad, dice, «comienza con el reconocimiento de dificultades que no se pueden resolver en el marco de formas de relación anteriores, e implica una renegociación y, a veces, una transformación en nuevas pautas de trato» (p. 148). Ya se ha mencionado que en ocasiones la primera dificultad está en el reconocimiento de que algo está cambiando, y la familia insiste en buscar una solución a la avería (la enfermedad, por ejemplo), por lo que la primera tarea para cada miembro de la familia consistirá en tomar en consideración cómo ha venido funcionando en su relación con los otros miembros del sistema, cuáles son sus necesidades, y las de los otros, y cómo quiere y puede participar en la nueva organización.

Muchas de las situaciones de conflicto que se reconocen en la práctica provienen de esta falta de negociación; el nuevo orden de funcionamiento es impuesto a una persona por el resto del sistema (uno de los hijos/as asume los cuidados en exclusiva, pudiendo provocar sobrecarga y ruptura del sistema de relación filial), o se acepta la situación sin evaluar las posibilidades, los recursos o los cambios que van aconteciendo (cuidar a un progenitor que siempre se ha vivido como demandante, con quien ha sido difícil la diferenciación, cuando en el aquí y el ahora el apego es más intenso). Todo ello puede además agravar la situación de los cuidados, no sólo para el cuidador, sino también para el anciano en la medida que no se prioriza qué es lo mejor para él. La situación que otra participante en el grupo ha vivido reproduce este conflicto: desde hace ocho años cuida de su madre, en su propia casa, dado que su hermano, cuenta, no ha planteado asumir esta tarea de manera compartida (viven ahora en localidades distantes). Relata además su experiencia de cuidado como una prolongación de lo que ha sido una demanda de atención constante de la anciana: durante años debía acudir ante la llamada de su madre si su padre llegaba bebido. Se trata de una situación que cada día la desborda más y que describe como:

No me he separado de ella ni de día ni de noche, hasta este año, después de ocho, ¡eh!: llévala al baño, dale de comer, dale conversación, ve a por medicinas, llévala al médico, ten cuidado que no se quiera levantar sola y se caiga [...]. Tiene un hijo, pero hasta este año que le he llamado y le he dicho: jo vienes a por tu madre, un mes, sólo te pido un mes, para poder ir a ver la tumba de mí marido, o te la llevo y te la dejo en la puerta de tu casa!

La cuidadora no se podía sostener en estas circunstancias, con su madre cada vez más incapacitada, sin red social en la localidad en la que viven y sin ningún apoyo familiar. Más cuando su capacidad de negociación con su único hermano estaba tan deteriorada que sólo le quedó amenazarle; el problema era que el instrumento de cambio era una anciana incapacitada y la solución final que se planteó fue un ingreso en residencia de esta mujer.

Como señalan Sison, Aparicio y Guinaldo (2012) todas las familias tienen su historia previa (modelos de funcionamientos, estilos de comunicación, normas y reglas) que han determinado las relaciones entre los miembros a lo largo de su vida como sistema, pero es ahora, cuando la dependencia aparece y hay que tomar decisiones, cuando se reactivan los viejos vínculos, las dificultades de diferenciación, las alianzas, etc. La cuidadora no había tenido oportunidad de negociar con su hermano, pero sí debía convivir con lo que para ella había sido la pauta familiar: estar sobreimplicada con su madre, no obtener reconocimiento y además no poder cuestionar, frente a ésta, la falta de implicación del hermano, quien había sido y era idealizado por la anciana como el hijo perfecto.

Por otro lado, la realidad de estas familias está caracterizada, en gran medida, por la ambigüedad, puesto que se encuentran atrapadas entre multitud de requerimientos, dudas, exigencias y cambios; entre una historia pasada y un presente que renueva experiencias, que obliga a tomar decisiones sobre otro, pero del que no pueden o no quieren desentenderse, una realidad sobre la que apenas se tiene control (¿cómo evolucionará la enfermedad?, ¿los cuidados que se dan son adecuados?), y en muchas ocasiones sin información socio-sanitaria precisa.

\subsection{Un duelo imposible}

Boss (2001) se refiere a una pérdida ambigua en aquellas situaciones en las que el ser querido está físicamente presente, pero ausente psi- 
cológicamente: «esta no es mi madre», nos decía una participante en el grupo. La autora añade que no está directamente relacionado el grado de severidad de la demencia del progenitor en los casos tratados con el estado depresivo de su cuidador, sino que es más bien el modo cómo éste percibe al enfermo, ausente o presente, lo que predice más acertadamente sus síntomas. Y sólo así se puede entender el motivo por el que dos mujeres del grupo «aconsejaban» continuamente a otra compañera, mientras ésta lloraba y se mostraba impotente frente a los síntomas de su madre, cómo debía sujetarla a la silla para que no se cayese, qué alimentación debía darle si ya no ingería sólidos, etc. Ninguna de las dos podía escucharla ni acoger el sufrimiento y la tristeza que aquella sentía en estos momentos, aún cuando ellas dos cuidaban de una anciana aún más deteriorada.

Esta ambigüedad se ponía de manifiesto continuamente en la experiencia del grupo de apoyo; aparecía tras expresiones de culpa, negaciones, impotencia, sentimientos de incompetencia, rechazo, pero también como humor, sentimientos de superación, reconocimiento de capacidades, oportunidades de resolver viejos dilemas, de reconciliación, etcétera. Es decir, la pérdida no sólo provoca situaciones de malestar, incertidumbre, agotamiento, desesperación, sino que se ofrece también como una oportunidad para el que cuida. El grupo, por tanto, se ha mostrado como un espacio donde plantear y afrontar estas contradicciones, asumir la impotencia, expresar el enfado, la rabia y todo ello desde la seguridad de no ser valorado y juzgado si no, por el contrario, comprendido y aceptado.

Es obvio que hasta este punto, se ha planteado la realidad, las vivencias, las dificultades de las familias que efectivamente aceptan atender a su familiar dependiente, precisamente el grupo iba dirigido a ellas, no se ha recogido ni analizado (quizá para un trabajo ulterior) qué ocurre cuando la familia no cuida, cómo entender el rechazo por el progenitor, la aparente falta de responsabilidad. En cualquier caso, como se ha tratado de exponer a lo largo de este trabajo, tratar de comprender las situaciones que viven las personas cuidadoras nunca debe implicar contemplarlas como una foto fija. Se trata, en suma, de hacer aún más compleja la situación para poder, paradójicamente, aceptar y resolver los dilemas a los que estas familias se enfrentan: aceptar la ambivalencia (querer lo mejor, pero no poder más, desear el final, por ejemplo), las continuas preguntas sin respuesta (¿por qué a ella?, ¿qué hicimos mal?, ¿hasta cuándo?), una situación cambiante en la que las soluciones nunca son perfectas y únicas ( si hasta ahora he podido cuidar en casa, ¿cuánto tiempo será esto posible?), la pérdida de control y la impotencia a veces (no puedo más, no sé qué hacer), buscar el reconocimiento pero no a cambio del sacrificio, pedir ayuda y reconocer los límites.

Unas últimas palabras de Siri Hustvedt, en su novela Elegía para un americano, resumen lo que en buena parte ha sido el objetivo de estas páginas:

Estuvimos hablando un rato sobre los sentimientos buenos y malos. Yo insistí en que tenemos ambos. Que tener malos sentimientos no hace que seas malo [...]. Sé que a veces lo que decimos es menos importante que el tono que usamos para decirlo. En todo diálogo hay una música, una armonía misteriosa y unas disonancias que vibran dentro del cuerpo como un diapasón.

\section{Referencias bibliográficas}

Abellán, A., Esparza, C., Castejón, P. y Pérez, J. (2011). Epidemiología de la discapacidad y la dependencia de la vejez en España. Gaceta Sanitaria, 25(s), 5-11. Disponible en: http://www.elsevier.es/es/revistas/gaceta-sanitaria-138/volumen-25/numero-sup12 Doi:10.1016/j.gaceta.2011.07.010 (12/04/2013).

Bazo, T. (2008). Personas mayores y solidaridad familiar. Politica y Sociedad, 45(2), 73-85.

Boletín Oficial del Estado. (2006). Ley 39/2006 de Promoción de la Autonomía Personal y Atención a las personas en situación de dependencia. BOE, 299, 44142-44156.

Boss, P. (2001). La pérdida ambigua. Barcelona: Gedisa.

Caillé, P. y Rey, Y. (1990): Había una vez...Del drama familiar al cuento sistémico. Buenos Aires: Nueva Visión

Camdessus, B., Bonjean, M. y Spector, R. (1995). Crisis familiares y ancianidad. Barcelona: Paidós. 
Carretero, S., Garcés, J., Ródenas, F. y Sanjosé, V. (2006). La sobrecarga de las cuidadoras de personas dependientes: análisis y propuestas de intervención psicosocial. Valencia: Tirant lo Blanch.

Casado, D., García-Gómez, P., López, A. y Massons, S. (2007). Empleo y apoyo informal entre las mujeres de mediana edad en España. En S. Jiménez-Martín (coord.), Aspectos económicos de la dependencia y del cuidado informal en España (pp. 33-67). Barcelona: Universidad Pompeu Fabra. Recuperado de http://www.imsersomayores.csic.es/documentos/documentos/fedea aspectos-01.pdf (consultado el 10/12/2012).

Durán, A. (2002). Los costes invisibles de la enfermedad. Bilbao: Fundación BBVA.

Gracia Fuster, E. (1997). El apoyo social en la intervención comunitaria. Barcelona: Paidós.

Hill, J., Fonagy, M., Safier, E. y Sargent, J. (2003). The Ecology of Attachment in the Family. Family Process, 42, 205-221.

Izquieta, J. L. (1996). Protección y ayuda mutua en las redes familiares. Tendencias y retos actuales. Revista Española de Investigaciones Sociológicas, 74, 189-208.

King, D. A. y Wynne, L.C (2004). The emergence of «Family Integrity» in later life. Family Process, 43, 7-21. doi: 10.1111/j.1545-5300.2004.04301003.x

La Parra, D. (2001). Contribución de las mujeres y los hogares más pobres a la producción de cuidados de salud informales. Gaceta Sanitaria, 15, 498-505., Disponible http://elsevier.es (Consultado 22 de enero de 2013).

Ministerio de Trabajo y Asuntos Sociales. Instituto de Mayores y Servicios Sociales. (2005). Libro Blanco sobre la atención a las personas en situación de dependencia en España. Madrid: Autor.

Navarro, J. (2004). Enfermedad y familia. Barcelona: Paidós.

Rogero, J. (2010). Los tiempos del cuidado. El impacto de la dependencia de los mayores en la vida cotidiana de sus cuidadores. Colección Estudio-Serie Dependencia No 12011. Madrid: IMSERSO.

Rolland, J. S. (2000). Familias, Enfermedad y Discapacidad. Barcelona: Gedisa.

Sison, A., Aparicio, A. y Guinaldo, A. (2012). Cuidando a los que cuidan: Los grupos de soporte psicológico. Mosaico, 53, 72-81.

Wynne, C. L. (1991). Un modelo epigenético de los procesos familiares. En C. Falicov (comp.), Transiciones de la familia (pp. 131-166). Buenos Aires: Amorrortu. 